\title{
Phosphoproteomics Illuminates Opioid Actions
}

\author{
Tao Che and Bryan L. Roth*10 \\ Department of Pharmacology, University of North Carolina School of Medical, Chapel Hill, North Carolina 27514, United States
}

\begin{abstract}
Opioids are widely used analgesic medications with a high potential for tolerance and dependence and represent a frequent cause of death due to overdose. Opioids mediate their actions via a family of opioid $\mathrm{G}$ protein coupled receptors. Elucidating the biochemical mechanism(s) responsible for both the therapeutic and deleterious side effects of opioids could provide a biochemical roadmap for selectively targeting therapeutic signaling pathways. Here we provide a perspective on emerging findings, which illuminate these signaling pathways via unbiased and quantitative phosphoproteomic analysis. What emerged from these studies is the discovery that certain deleterious actions mediated by the $\kappa$ opioid receptors appear due to specific activation of mTOR pathways. The findings imply that designing drugs, which bypass mTOR signaling, could yield safer and more effective analgesics.
\end{abstract}
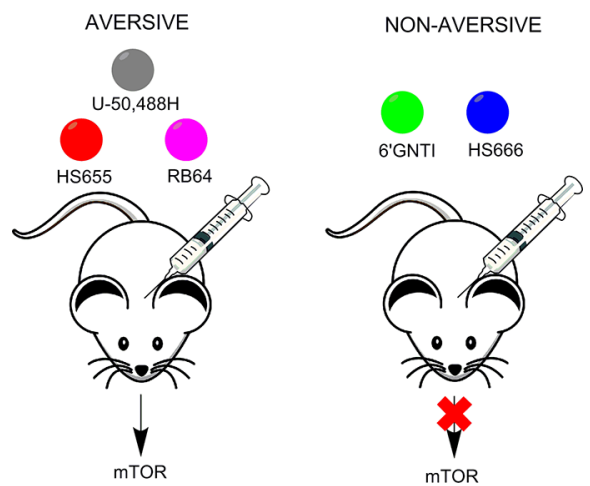

$\mathrm{O}$

pioid drugs like morphine and oxycodone are powerful painkillers that produce analgesia by activation of opioid G-protein coupled receptors (GPCRs). Although opioids are quite useful for alleviating pain, they can also elicit an array of harmful side effects, such as aversion, hallucinations, addiction, and death by respiratory depression. Thus, a new class of nonopioid analgesics could go a long way toward alleviating the prevailing opioid crisis. Alternatively, could we develop a biased or functionally selective opioid that preferentially produces desired effects and avoids undesired effects? ${ }^{1,2}$ Currently, there are many comprehensive cell-based assay platforms (proximity, distribution, or signaling assay) available to study biased agonism in vitro. ${ }^{3}$ However, the in vitro pharmacology is frequently inconsistent with the in vivo behavioral activities due to the complexity of signaling networks in vivo. How to directly measure drug actions and GPCR activities in real-time in vivo remains an underutilized approach.

In a breakthrough study, Liu et al. $^{4}$ applied an optimized mass spectrometric approach to quantify phosphorylation events in an unbiased way in the brain after drug administration. Protein phosphorylation is considered to be one of the most abundant as well as one of the most important post-translation modifications for signaling networks. The $\kappa$ opioid receptor (KOR) was used as a model system along with five KOR ligands (U-50,488H, 6'GNTI, RB64, HS665, and HS666), which display differential in vitro and in vivo pharmacology. In particular, two of the drugs, 6'GNTI and HS666, do not induce the classical KOR-mediated aversion that has been observed for U-50,488H, RB64, and HS665.

After administration of these five KOR agonists, Liu et al. analyzed the phosphoproteomic architecture from five brain regions (striatum, cortex, hippocampus, medulla oblongata, and cerebellum), each having different levels of KOR expression. The authors identified more than 50000 phosphorylation sites after stimulation, and in combination with the use of dynorphin- and KOR-knockout mice, they identified the phosphorylation sites uniquely related to KOR activation. The authors determined that the dynamic changes in KOR phosphorylation displayed spatio-temporal control. Further analysis of KOR-mediated phosphorylation identified those patterns relevant to specific neuronal circuits, in particular, dopamine-, glutamate-, and $\gamma$-aminobutyric acid (GABA)-ergic signaling, thereby providing a connection between KOR activation and signaling in these neurons.

They also explored the phosphorylation patterns between pharmacologically different ligands. U-50,488H and 6 'GNTI, for instance, only shared $30-50 \%$ of the specifically identified sites. Here we list some of the more interesting detailed changes found after KOR activation by U50,4088H:

1) Phosphorylation of the extracellular signal-regulated kinase ERK1 $\left(\mathrm{Thr}^{203}\right.$ and $\left.\mathrm{Tyr}^{205}\right)$, the cannabinoid receptor $1\left(\mathrm{CB} 1, \mathrm{Ser}^{317}\right)$, the GABA-B receptor $\left(\mathrm{Ser}^{762}\right)$, the $G$ protein $\gamma$ subunit $12\left(\operatorname{Ser}^{7}\right)$, and the NMDA receptor 2a $\left(\operatorname{Ser}^{1459}\right)$

2) Dephosphorylation of dopamine- and cyclic adenosine monophosphate-regulated neuronal phosphoprotein (DARPP-32, $\mathrm{pSer}^{97}$ ) and the kinase Src $\left(\mathrm{pSer}^{17}\right)$.

This observation of many dephosphorylation events implies that both phosphatases and kinases are involved in the synaptic signaling transduction pathways utilized by opioids.

Interestingly, some of these phosphorylation/dephosphorylation events were brain-region specific. Dephosphorylation of DARPP-32 exclusively occurred in the striatum and was specific to $\mathrm{Ser}^{97}$, while phosphorylation of ERK1 was only observed in the hippocampus. All of these could be physiologically important because they play essential roles in

Received: July 31, 2018

Revised: August 29, 2018

Published: September 4, 2018 
regulating synaptic plasticity. Importantly, $6^{\prime}$ GNTI and HS666, which do not produce aversion in vivo, did not regulate these sites, which implies that these phosphorylation events are linked to this serious side effect of KOR activation.

The authors next focused on revealing the detailed pathways that may be responsible for aversive behavior in vivo. Through extensive bioinformatic analysis of signaling pathways regulated only by U-50,488H, HS665, and RB64, the mechanistic target of the rapamycin (mTOR) signaling pathway appeared to be the most important. To determine whether the mTOR pathway is related to the aversive actions of these drugs, the mTOR-specific inhibitor temsirolimuc was applied followed by $\mathrm{U}-50,488 \mathrm{H}$, a KOR agonist that produces profound aversion. As predicted, pretreatment with an mTOR inhibitor abolished a measure of aversion (conditioned place aversion) in mice. In addition, the mTOR inhibitor had no effects on U-50,488Hmediated anticonculsive and antinociceptive effects, suggesting that mTOR pathways are involved in the side effects but not therapeutic actions of KOR opioids. These results imply that the desired (antinociception) and undesired (aversion) effects can be pharmacologically separated, at least for KOR opioids. However, whether the aversion effect, or particularly the mTOR pathway, is mediated by classical arrestin activation still requires further research. A parallel study on nalfurafine (a nonaversive drug at KOR)-mediated phosphoproteomic events indicated that it does not activate the mTOR pathway either. ${ }^{5}$ Interestingly, nalfurafine was shown as a balanced KOR agonist, which equally activates both the $G$ protein and the arrestin pathway in vitro, ${ }^{5}$ but whether it could activate the arrestin pathway in vivo is unknown.

This work provides an exemplar of how unbiased phosphoproteomics can reveal signaling pathways involved in both the therapeutic and deleterious actions of KOR opioids. The results also support the potential for combination therapy (opioid plus mTOR inhibitor) as a promising approach for pain therapeutics, although current mTOR inhibitors elicit immunosuppressive side effects (Figure 1). This high-

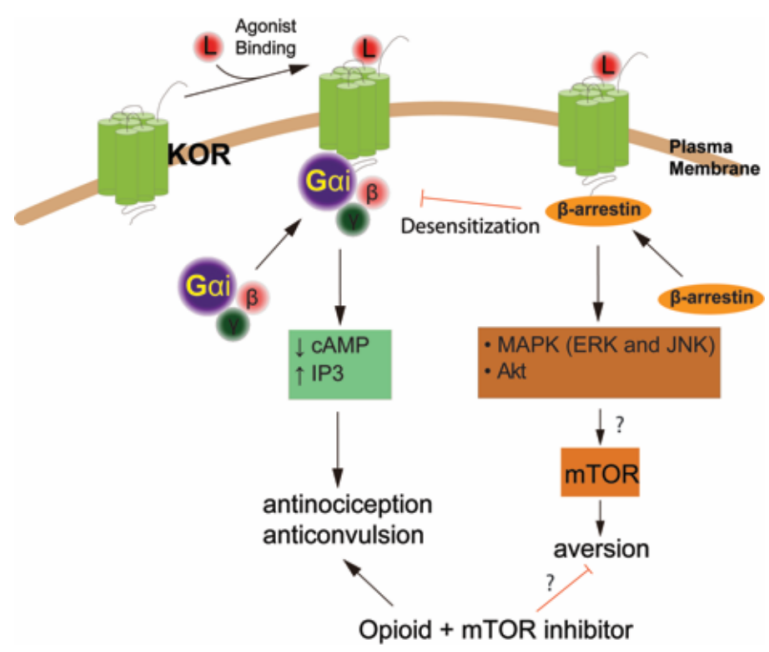

Figure 1. Model of KOR agonist-mediated downstream signaling.

throughput phosphoproteomic approach could be extended to study downstream events of other GPCR members and other families of proteins and thereby illuminate many of the signaling events relevant to drug actions in the brain.

\section{AUTHOR INFORMATION}

\section{Corresponding Author}

*E-mail: bryan_roth@med.unc.edu. Phone: 919-966-7535.

ORCID $\odot$

Bryan L. Roth: 0000-0002-0561-6520

Funding

This work was supported by grant DA035764 from the National Institute on Drug Abuse.

Notes

The authors declare no competing financial interest.

\section{ACKNOWLEDGMENTS}

Dr. Brian K. Krumm is gratefully acknowledged for helpful discussions and comments.

\section{REFERENCES}

(1) Manglik, A., Lin, H., Aryal, D. K., McCorvy, J. D., Dengler, D., Corder, G., Levit, A., Kling, R. C., Bernat, V., Hubner, H., Huang, X. P., Sassano, M. F., Giguere, P. M., Lober, S., Da, D., Scherrer, G., Kobilka, B. K., Gmeiner, P., Roth, B. L., and Shoichet, B. K. (2016) Structure-based discovery of opioid analgesics with reduced side effects. Nature 537, 185-190.

(2) Che, T., Majumdar, S., Zaidi, S. A., Ondachi, P., McCorvy, J. D., Wang, S., Mosier, P. D., Uprety, R., Vardy, E., Krumm, B. E., Han, G. W., Lee, M. Y., Pardon, E., Steyaert, J., Huang, X. P., Strachan, R. T., Tribo, A. R., Pasternak, G. W., Carroll, F. I., Stevens, R. C., Cherezov, V., Katritch, V., Wacker, D., and Roth, B. L. (2018) Structure of the Nanobody-Stabilized Active State of the Kappa Opioid Receptor. Cell 172, 55-67.e15.

(3) Smith, J. S., Lefkowitz, R. J., and Rajagopal, S. (2018) Biased signalling: from simple switches to allosteric microprocessors. Nat. Rev. Drug Discovery 17, 243-260.

(4) Liu, J. J., Sharma, K., Zangrandi, L., Chen, C., Humphrey, S. J., Chiu, Y. T., Spetea, M., Liu-Chen, L. Y., Schwarzer, C., and Mann, M. (2018) In vivo brain GPCR signaling elucidated by phosphoproteomics. Science 360, eaao4927.

(5) Liu, J. J., Chiu, Y. T., DiMattio, K. M., Chen, C., Huang, P., Gentile, T. A., Muschamp, J. W., Cowan, A., Mann, M., and Liu-Chen, L. Y. (2018) Phosphoproteomic approach for agonist-specific signaling in mouse brains: mTOR pathway is involved in kappa opioid aversion. Neuropsychopharmacology. 